\title{
Segregación Social y Políticas Públicas: Un Estudio sobre el Turismo en la isla Mexcaltitlán, México
}

\section{Social Segregation and Public Policies: A Case Study About the Tourism in Mexcaltitlán Island}

\author{
Sara Conceição Paula \\ Mestranda em Ciência Econômicas e Sociais pela Universidad Autónoma de Sinaloa, México, sara.angrense@gmail.com \\ http://lattes.cnpq.br/7359491747438256
}

Fabíola Cristina Costa de Carvalho

Doutoranda em Ciências Sociais pela Universidad Autónoma de Sinaloa, México, fabiolacarvalho.tur@hotmail.com http://lattes.cnpq.br/8325615691511449

Resumen: El objetivo de este trabajo es problematizar la conformación de espacios de segregación como efecto de los fundamentos de la política pública. Específicamente, se observa las incoherencias del Programa de Pueblos Mágicos a partir de la experiencia de intervención del turismo en la isla Mexcaltitlán de Uribe a partir de un análisis del fundamento de las políticas públicas basada en la igualdad de oportunidades y asistencia condicionada y focalización. La investigación que utiliza una revisión bibliográfica, datos secundarios y observación directa identificó un proceso de segregación política formalizando la (no) intervención gubernamental, además de una evasión del patrimonio cultural. En el lugar de una acción supuestamente articulada por el Estado, la isla fue rechazada (sobrepenalizada) por sus propias condiciones de vulnerabilidad social, económica y estructural.

Palabras clave: Estado, marginalización social, Pueblos Mágicos, México

Abstract:The aim of this paper is to discuss about the creation of spaces for segregation as a result of the prospects of public policies. Specifically, we observed the inconsistencies of the Magical Towns Program from the experience of intervention of tourism in the island Mexcaltitlánde Uribe by analyzing the basis for public policies based on equality of opportunities and conditional assistance and focusing. The research, which is based on literature review, secondary data and direct observation, identified a process of political segregation formalizing the non-governmental intervention, in addition to an evasion of cultural heritage. In place of an allegedly articulated action by the State, the island was rejected (sobrepenalizada) by its own terms of social, economic and structural vulnerability.

Keywords: State, social marginalization, Magical Towns, Mexico

Texto completo em português: http://www.apgs.ufv.br Full text in Portuguese: http://www.apgs.ufv.br

\section{Introducción}

El objetivo de este artículo es problematizar la conformación de espacios de segregación intensificados por cambios en las perspectivas de la política pública. Específicamente, se observa las incoherencias del Programa de Pueblos Mágicos a partir de la experiencia de intervención del turismo en la isla Mexcaltitlán de Uribe, en Nayarit, México.

La crisis en la perspectiva del Estado de Bienestar social cuanto a su carácter universal introduce una serie de retos con respecto a la vulnerabilidad social o expansión de la vulnerabilidad (Castel, 2014), principalmente en el contexto de países no desarrollados. Por el escenario de dependencia, además de las instituciones debeles (North, 2012, p. 1993), el principio de fundamentación del mercado, al contrario de los cambios propuestos, no fue capaz de asegurar que la expansión del comercio internacional actuase como motor del crecimiento que aseguraría la dinámica de la economía reforzando una competitividad a nivel internacional (Moreno-Brid \& Bosch, 2014). Tales limitaciones ofreció espacio a una sociedad fragmentada que ejercita la segregación en diversos ámbitos. Mientras efectiva una responsabilización individual en el sustento de la reducción del Estado en el carácter universal del derecho, pero "máximo" en el quesito de apoyo a los grandes capitales (Wacquant, 2011; Boltvinik, 2013). Ese cambio de perspectiva, identificado esencialmente en un proyecto económico, permea distintos aspectos sociales involucrando la producción social (Montangut, 2004).

Presentase como argumento central que el modelo de intervención de la política analizada parte de una lógica de actuación de transferencias condicionadas orientada hacia al mercado, de carácter inmediatista y exploratorio del turismo.Todavía se considera que esta situación puede resultar en una marginalización de los territorios reproduciendo una sociedad fragmentada. Los efectos de este escenario se manifiestan mínimamente en las esferas: (a) la ausencia de suporte y política estatal que ofrezca una posibilidad de desarrollo a la comunidad local frente insipiente iniciativa privada; $y$, (b) un rechazo con relación a un área de importante relevancia histórica, sumado a laextracción del patrimonio cultural de la localidad; y (c) el destaque de la culpa de la comunidad local frente al retiro del beneficio público - en el caso, el título de Pueblos Mágicos-,

Correspondência/Correspondence: Sara Conceição Paula. Universidad Autónoma de Sinaloa, Prolongación Josefa Ortiz de Domínguez S/N, Cd Universitaria, 80040 Culiacán Rosales, Sin., México.

sara.angrense@gmail.com 
destacando la insuficiencia de méritos individuales de la comunidad local.

La pertinencia es la discusión de las contradicciones del actual modelo de intervención en espacios turísticos en contraste al fenómeno de segregación económica y social. Conforme observado en la literatura, la creación de ciudades producto, fundamentado en el mercado y la creencia de resultados lineares, tienden a una potencializar resultados destructivos para la comunidad local, con destacados espacios de segregación, privatización del patrimonio público operando crecimiento, pero tendenciosa a una estructura social polarizada. Se destaca que este paper se presenta las consideraciones parciales de una investigación en curso que tiene como objetivo analizar cómo los fundamentos de la política social refleten el retorno de la "racionalidad penal moderna" a partir de las prácticas de transferencias condicionadas y la ebullición de la estructura penal en Brasil y México a partir de 1990.

Para llevar a cabo la discusión propuesta el trabajo está estructurado en tres partes, además de esta introducción y del apartado final en el que se presenta las consideraciones parciales de la investigación. En el segundo apartado se presenta los cambios de fundamentos en las políticas públicas pos neoliberalismo. El tercer, describiese la metodología. En secuencia se expone las principales características del Programa Pueblos Mágicos (PPM) y su lógica de segregación por una transferencia condicionada. Siguiendo, se destaca los efectos de la intervención del PPM en la isla Mexcaltitlán de Uribe, en México. Como resultado, se identificó que hubo un proceso de segregación política, que formalizó la (no) intervención gubernamental además de una evasión del patrimonio cultural local. En el lugar de una acción supuestamente articulada por el Estado conforme presentado en la elaboración del programa la isla fue rechazada por sus propias condiciones de vulnerabilidad social, económica y estructural.

\section{Igualdad de posiciones y la Vulnerabilidad Social}

Es posible identificar una interpretación sociológica del fenómeno de la asistencia estatal a partir del análisis de la crisis del modelo de igualdad de posiciones en favor del modelo de igualdad de oportunidades basado en la meritocracia (Vite Pérez, 2014). De acuerdo con Dubet (2012) tanto la teoría de igualdad de posiciones como la teoría de la igualdad de oportunidades se basan en la concepción de justicia social, proponiendo disminuir las contradicciones presentes en la sociedad, a partir del principio de que todos los individuos son semejantes, todavía viven en sociedades desiguales. La primera teoría propone la reducción de la distancia entre las posiciones sociales, de forma que los ricos sean menos ricos y los pobres menos pobres. Mientras tanto, la segunda teoría consiste en el argumento de que todos los individuos deben tener la posibilidad de alcanzar la misma posición. Conformada por la necesidad de expansión del capital, hay una ampliación de tendencias a la mercantilización de los servicios básicos de seguridad social (Esping-Andersen, 1993).
Así, cada vez más residual, la política social, que en su análisis no se puede distanciar de la política económica (Montagut, 2004) así como la estructura punitiva-, tiene aspectos elementares para una conformación de tendencias que indica una reestructuración de la sociedad de trabajo.

Asumida y articulada en forma de política pública, la individualización de la responsabilidad, se establece en el proceso de construcción de un Estado ideal que respalda una "responsabilidad individual irrestricta", mientras posee una irresponsabilidad colectiva (política) (Wacquant apud Abramovay, 2010 , p. 12). El efecto de la disminución de las funciones del Estado. Respaldada por el fundamento de igualdad de oportunidades, la actuación se restringe a intervenir para el amparo de segmentos en vulnerabilidad social condicionados a una determinada respuesta.

De esa manera, en los países donde la responsabilidad por el éxito se basa en la meritocracia individual, surge la creación de dispositivos que tienden a condicionar el derecho a la asistencia, resultando en un proceso de restricción o barreras de desamparo social, mientras se ha compartido la realidad de la precariedad laboral. Esta dinámica reflete la crisis de la sociedad del trabajo, al paso que incorpora un desmantelo de los derechos sociales que ha consolidado una cosmovisión que comprende la desigualdad social consonante a los méritos individuales (Abramovay, 2010; Wacquant, 2011; Vite Pérez 2014) postulando como natural la desigualdad socialmente producida.En esto cambio de fundamento de las políticas públicas pos neoliberalismo, hay una tendencia al atendimiento asistencial cada vez más extremado y selectivo (Boltvinik, 2013), o a proyectos que ofrezcan retornos a corto plazo y rentabilidad fuertemente sustentados por iniciativa privada (Hernández López, 2009,p. 411). Al investigar la pobreza en México y destacar la necesidad de "reformar la reforma" de las políticas sociales, Boltvinik $(2012,2013)$ presenta una crítica a las diferentes formas de medición de la pobreza y los intereses envueltos en cambios en estas mediciones por el Consejo Nacional de Evaluación de la Política de Desarrollo Social (Coneval). Según el autor, el programa de medición de la pobreza utilizado por Coneval en México - intersección de los conjuntos2 (2012) - nunca antes se había usado en América Latina y tiende a considerar apenas la población en extrema pobreza. La justificativa metodológica para la escoja del método es inexistente y objetiva reducir la incidencia de la pobreza intensificada en el país3.

A pesar de la vulnerabilidad social ser un efecto considerablemente grave, el fundamento de los méritos individuales también consubstancia la "racionalidad penal moderna" que para Abramovay (2010), es la base tanto del

\footnotetext{
1 Véselo en Hernández López (2009) el estudio sobre la implementación de PPM en el Pueblo Tequila.

2Véselo en Boltvinik (2012, p. 28)

${ }^{3} \mathrm{El}$ autor también resalta la fuerte influencia internacional en Coneval. Principalmente de Alkire y Foster de la Iniciativa de Pobreza y Desarrollo Humano de la Universidad de Oxford, y las propuestas del Programa de Naciones Unidas para el desarrollo (PNUD) en Nova York. Semejantemente, ocurre una tendencia entre órganos internacionales reguladores, como el Banco Central y el PNUD que compiten para "minimizar" la pobreza mundial.
} 
Contracto Social cuanto del Derecho Penal. Esta expresión representa la creencia enaltecida en el individuo edificado sobre la consciencia de sus facultades y responsabilidades; enaltece los hechos individuales mientras penaliza los "caracteres" incapaces como "sucios, feos y peligrosos"4.

Para Wacquant (2012) el welfarestate, es sustituido por dos "nuevas"5 perspectivas de actuación del Estado frente la vulnerabilidad social: (a) workfare, que representa las ciénagas o un acceso condicionado a la asistencia social otrora asumida como derecho; condicionado a sumisión a un trabajo precario y, (b) prisión fare correspondiendo a la postura estatal de prender y segregar, sea por medio de la prisión-edificio por espacios que duplican la lógica de segregación. Ese proceso es concomitante a un incremento significativo en los niveles de encarcelamiento (International Centre for Prision Studies of King's College London ICPS, 2014), ocasionando una crisis en el sistema penal, principalmente en países sobe influencia de un Welfare State residual, lo que fornece a América nueve puestos entre el ranking de los doce países con las mayores tasas de encarcelamiento por habitantes

En México, conforme destacada por Jusidman (2012, p. 362) se ha identificado prácticas de discriminación y segregación tanto en los servicios sociales cuanto en la ubicación en el territorio. Para la autora, el padrón de crecimiento urbano modelo en México "aísla, separa y propicia conflictos y tensiones, donde los más afectados son los pobladores urbanos pobres".Estos espacios reciben titulaciones basadas en la fragmentación moral de una sociedad tomada al pánico para aniquilar o alejar "monstros" en los cuales están el bandido, la prostituta, narcos, terroristas, sucios, flojeras, favelas, el indio, el negro entre otros identificados en el imaginario colectivo (Martínez, 2010).

Las discusiones de los efectos de programas de turismo comprendidos bajo esta perspectiva pueden reflexionar sobre las contradicciones en el incremento de la desigualdad social en estas áreas. Así se observa la creación de espacios productos que segregan los actores locales de la actividad, como también perdidas de la identidad local y privatización patrimonial.

\section{Metodología}

Para alcanzar el objetivo de problematizar la conformación de espacios de segregación, este artículo utiliza una revisión bibliográfica, datos secundarios y observación directa con el fin de identificar las incoherencias del Programa de Pueblos Mágicos a partir de la experiencia de intervención del turismo en la isla

${ }^{4}$ Aquí se hace referencia a la investigación de Teixeira y Matsuda (2012) sobre la población en situación de calle. (VéseloenTeixeira y Matsuda. (2012). Feios, sujos e malvados. Controle Policial.Le Monde Diplomatique Brasil.Edição 56.)

${ }^{5}$ Es importante destacar que la literatura ya apunta la actuación coercitiva del Estado en cuanto fundamento predominante en los períodos anteriores al Estado de Bienestar social a fin de configurar el mercado de trabajo. Uno de los estudios más destacados sobre configuraciones de mercado de trabajo y punición fue de Georg Rusche y Otto Kirchheimer (vérselo Rusche \& Kirchheimer, 2004). Tales investigaciones pueden indicar que el consenso de welfarestate puede remontar a un gran paréntesis en la historia del capitalismo que tiende a cerrarfrente a períodos de crisis.
Mexcaltitán de Uribe, en Nayarit, México. Para articular el PPM con la asistencia condicionada y la segregación, la investigación presenta en un primer momento el análisis del PPM a partir de los documentos que articulan planeamiento, presentación del programa y practicas operacionales de incorporación y permanencia. En seguida, se destaca algunos detalles de la isla y sus contrastes. A posteriori, se presenta la experiencia de intervención y los desencadenamientos en la isla de Mexcaltitán de Uribe del período de 2001 (cuando fue declarada Pueblo Mágico por el Gobierno Federal) hasta 2009 año en que se ha retirado el título de pueblos mágicos (mismo siendo considerada "zona de monumentos históricos" desde 1986).

\section{Pueblos Mágicos y la intervención en Mexcaltitán}

\subsection{El programa Pueblos Mágicos y sus configuraciones}

Las organizaciones internacionales tienen un rol decisivo en la normalización y en la innovación de los servicios turísticos ofrecidos a nivel mundial, particularmente desde la década de 1970, cuando empezaron a influenciar más fuertemente las tendencias del mercado de viajes en el mundo, aproximando las políticas públicas relacionadas con el turismo. En la esfera pública las agencias de desarrollo internacional también favorecen el desarrollo del turismo en muchos países en desarrollo, mientras contribuyen a la difusión de técnicas y métodos de la planificación y gestión turística (Carvalho, 2014).

En este escenario, ajustado a los direccionamientos de la Organización Mundial de Turismo (OMT) para el desarrollo, específicamente con respecto a las investigaciones que están relacionadas con la importancia económica del turismo, las políticas de estímulo a la competitividad se volvieron en un eje central de los países de América Latina.

Así en el inicio del siglo XXI el turismo latinoamericano ha buscado ampliar y diversificar sus fuentes de atracción, por ejemplo, por la línea de la cultura y de la historia (BalslevClausen\& Alberto-Velázquez, 2013). En ese escenario, el Programa Pueblos Mágicos (PPM) nació en el 2001 como una estrategia de desarrollo turístico, fundada por la estructuración de una oferta complementaria y diversificada hacia el interior del país, asentada en atributos históricos y culturales de localidades singulares (México, 2011a).

Un Pueblo Mágico se caracteriza como una localidad que "tiene atributos simbólicos, leyendas, historia, hechos trascendentes, cotidianidad, en fin magia que te emanan en cada una de sus manifestaciones socio-culturales" (México, 2015a6).

De acuerdo con Balslev-Clauseny Alberto-Velázquez (2013) el PPM incorporó 30 localidades en todo el país, en 2001 y, en el 2011, se había completado 48 pueblos al programa, mientras que, desde fines del 2012,se han completado 83 pueblos participantes del programa.

${ }^{6}$ Véaselo en http://www.sectur.gob.mx/pueblos-magicos/\#prettyPhoto Consultado en 05 mayo 2015. 
Con respecto a la implementación del Programa en nivel nacional, en la "Meta IV.4. México Próspero", del Plan Nacional de Desarrollo 2013-2018 (PND) se establece el Objetivo 4.11, sobre el aprovechamiento del potencial turístico de México, la Estrategia 4.11.2 acerca del establecimiento de la diversificación e innovación de la oferta turística, la consolidación de destinos turísticos y el desarrollo de segmentos poco impulsados en el país(México, 2013, p. 143).

Además en el Programa Nacional de Infraestructura 20142018(PNI), publicado en el Diario Oficial de la Federación, en 29 de abril del 2014, su Estrategia 6.1establece como una línea de acción la reconversión de la infraestructura e imagen urbana ${ }^{7}$ de Pueblos Mágicos, con el fin de consolidar una oferta turística atractiva para el país (México, p. 2014).

Por lo tanto se observa que uno de los objetivos del programa, sino el principal, está centrado en la diversificación de la oferta turística de México, con base en la intervención física de los destinos, o sea, la construcción de infraestructura. Es decir, considerase que las poblaciones que sufren la intervención de la política por medio del PPM requieren orientarse para fortalecer y optimizar el aprovechamiento racional $^{8}$ de los recursos $y$ atractivos naturales y culturales, el fortalecimiento de la infraestructura, de la calidad de los servicios, de la innovación y del desarrollo de acciones que contribuyan a saltar el crecimiento del mercado turístico (México, 2015a).Cuanto al proceso, de presenta que:

[Así que] en el 5 de junio de 2002 se suscribió un Convenio General de Colaboración celebrado entre la Secretaría de Turismo y la Secretaría de Desarrollo Social; Secretaría de Educación Pública; Secretaría del Trabajo y Previsión Social; Secretaría de Economía; Secretaría de Medio Ambiente y Recursos Naturales; Fondo Nacional para el Fomento de las Artesanías; Consejo Nacional para la Cultura y las Artes; Banco Nacional de Obras y Servicios; Comisión Federal de Electricidad; Comisión Nacional del Agua, e Instituto Nacional de Antropología e Historia; el cual tiene por objeto establecer las bases de colaboración entre las partes, para apoyar la elaboración, desarrollo, cooperación, ejecución y evaluación de los logros del Programa Pueblos Mágicos; por lo que es relevante la participación de dichas dependencias e instituciones gubernamentales en el proceso de permanencia al Programa Pueblos Mágicos, como integrantes del órgano de apoyo intersecretarial que al efecto se conforme (México, 2011a, p. 1).

Es importante mencionar que para la ejecución del programa, por lo menos en el nivel de los documentos institucionales, se propone la estructuración de un trabajo conjunto y multisectorial entre diversas secretarias y esferas del gobierno.

Cuanto la incorporación de una localidad al programa requiere la existencia de atractivos turísticos diferenciados, es decir, elementos arquitectónicos, históricos, edificios, fiestas, tradiciones, productos artesanales, cocina tradicional que compartan del "imaginario colectivo de la nación" (Pueblos Mágicos reglas de operación, 2012 apud, Balslev-Clauseny Alberto-Velázquez, 2013).De acuerdo con México (2011a) para mantener el nombramiento de Pueblo Mágico la localidad debe cumplir los requisitos:

I. Mantenimiento y funcionamiento de un Comité Pueblo Mágico con seguimiento de acuerdos;

II. Aprobación y punto de acuerdo del Congreso del Estado, donde se establezcan los recursos presupuestarios por asignarse al Pueblo Mágico;

III. Cumplimiento de planes, programas y normativa;

IV. Fortalecimiento e innovación del catálogo de productos turísticos (servicios certificados);

V. Funcionamiento y adecuación de los servicios de salud y de seguridad;

VI. Evaluación del impacto en el desarrollo turístico;

VII. Mantenimiento de las relaciones comerciales con al menos un intermediario de servicios turísticos;

VIII. Contar con un Sistema de Información Estadística;

IX. Integración de un informe detallado de actividades (anual), y

$X$. Seguimiento a otros elementos que considere la Secretaría como relevantes para la operación del Programa Pueblos Mágicos.

Además, para ingresar al programa, las localidades deben: 1) estar ubicadas cerca de centros turísticos importantes; 2) estar a un radio de influencia no mayor a una hora de distancia tiempo $(200 \mathrm{~km})$ de un gran destino turístico que lo soporte; 3) que el turismo sea una prioridad en los instrumentos de planeación municipal; 4) existir (o haber el compromiso de elaborarse) un plan urbano turístico; y 5) contar con una Agenda 21, además de una población base de 20000 habitantes (Sectur, 2010; BalslevClausen\& Alberto-Velázquez, 2013).

\subsection{PPM y la igualdad de oportunidades}

Como presentado, el objetivo del Programa Nacional de Turismo (2001-2006) con la creación del PPM fue apoyar el desarrollo turístico sustentable en localidades que poseen atributos de singularidad y que serían representadas por una marca de exclusividad y prestigio delante de su valor atractivo.

Entretanto, en el documento "Guía de Incorporación y Permanencia Pueblos Mágicos" (México, s/d), se presenta un fuerte direccionamiento a la creación de ciudades productos utilizando la actividad turística para la captación de divisas con la orientación de atender "las motivaciones y necesidades de los viajeros".

El documento plantea una coordinación social, interinstitucional e intergubernamental juntamente con la iniciativa privada para privilegiar la participación de la comunidad receptora.

Cada Pueblo Mágico debe atender y monitorear la sustentabilidad de la localidad, con base a indicadores básicos que permitan evaluar el comportamiento de la actividad turística con el apoyo de sus autoridades estatales y con la orientación de la Secretaría de Turismo, así como atender los resultados y recomendaciones derivadas de los Diagnósticos de Competitividad y Sustentabilidad de los Pueblos Mágicos (México s/d, p.6).

En esta etapa, distinguiese la comunidad "tanto la directamente relacionada con la actividad turística y negocios afines" de la sociedad civil (México s/d, p. 6). Aquí se postula "una alianza (Gobernanza) entre los tres niveles de gobierno, la sociedad local y los actores económicos, empresas y 
organizaciones, que deberá trascender administraciones gubernamentales" (México s/d, p.7). Como etapas de este proceso, el PPM postula diversas articulaciones - que no corresponden a los objetivos del programa, pero son contempladas en los criterios de incorporación y permanencia en el programa - donde diferentes órganos gubernamentales se articulan con el fin de promover el desarrollo turístico de forma integral.

El programa se caracteriza más como una política incremental, que busca una solución a corto plazo para el desarrollo de los pueblos que no son incorporados en otras acciones para el desarrollo turístico. Todavía su lógica de acción puede manifestar una discapacidad con respecto a la atención de las demandas sociales específicas de las regiones más frágiles, del punto de vista estructural y socioeconómico. Como consecuencia de la falta de recursos humanos, administrativos y culturales, que son necesarios para el éxito de alcance de los objetivos del programa, se puede generar un efecto contrario, de intensificación de los problemas sociales.

Es importante identificar que los criterios de incorporación y permanencia del programa Pueblos Mágicos", no presenta, en su objetivo general o específico, proteger y guardar la riqueza cultural. Además, no hay claramente objetivos que se relacione con el desarrollo social de la comunidad. Así, la utilización de términos amplios y genéricos en los objetivos específicos fragilizan la elucidación del papel del programa para los destinos que son incorporados, como también torna ambigua la verificación de las metas y de cómo serán evaluadas las acciones llevadas a cabo. En ese sentido, los objetivos tales como "Provocar un mayor gasto en beneficio de la comunidad receptora", a pesar de que se aproximan a los términos de apoyo a la comunidad local, es general e impreciso, de manera que no esclarece al menos quien es esta "comunidad receptora"(Hernández López, 2009, p. 419).

De igual forma, las expresiones para utilización del programa, son dispuestos seis actividades importantes de operación: (1) Inversión pública para favorecer el desarrollo turístico local; (2) Desarrollo e innovación de productos turísticos; (3) Certificación turística; (4) sustentabilidad turística; (5) coordinación social, interinstitucional e intergubernamental; (6) Mercadotecnia Integral. Es posible identificar en el documento que ninguno de ellos posee un direccionamiento al desarrollo social, mientras que la sustentabilidad planeada está relacionada con sostener la actividad turística, y no necesariamente preocupase con la inclusión de la comunidad local en un producto turístico endógeno.

Otro contraste en el documento es que los objetivos se orientan por la actuación del sector privado como un elemento único para la estimulación del mercado turístico. El PPM ofrece una "oportunidad" de actividad productiva. Esta, por su vez, baliza dos articulaciones. La primera es la orientación al fundamento de mercado rumo a iniciativa privada. La segunda es la responsabilización de la "sociedad civil" y del gobierno local

${ }^{9}$ Véaselo en Hernández López (2009) el estudio sobre la implementación del PPM en el Pueblo Tequila. mientras se acota el papel del Estado que, a pesar de verticalizador de la política, inhibe la elaboración de mecanismos para ofrecer las mismas posiciones a las ciudades a cual se destina el "desarrollo del turismo sostenible".

Las consecuencias de explicar las desigualdades sociales partir de la meritocracia, una vez que la responsabilidad por el fracaso o éxito recae sobre el individuo (en el caso, en la comunidad), indica una imposibilidad o un ejercicio exhaustivo comprender el Estado en cuanto aparato capaz o con la función de universalizar derechos delante las incapacidades y conflictos históricamente identificadas. De esa forma, los distintos actores, que evidentemente no parten del mismo posicionamiento, sufren severos prejuicios conducido por las dinámicas de poder en el mercado.

Considerando un programa que favorece la iniciativa privada, ignorase las distintas posiciones de la comunidad local delante los agentes con mayores capitales, ejercitando así la conformación de espacios de segregación. Es decir, el fundamento de la igualdad de oportunidades ofrece espacio para la conformación de otro fenómeno, caracterizado por la estratificación y segregación social. La perspectiva hacia al privado desencadena una segregación del habitante local en favor de las ciudades productos, donde "se ocultan las necesidades y las condiciones reales de vida de la gente" (Hernández López 2009, p. 41).

Además, Hernández López (2009) destaca una pérdida de la identidad local a favor de los mercados competitivos estandarizados, que impulsan la privatización y no la patrimonización de la cultura. Un turismo de elite dirigido a un tipo de cliente mejor atendido con empresas ya competitivas, sobrepujando los negocios locales.

\subsection{Isla de Mexcaltitán de Uribe y sus contrastes}

Mexcaltitán de Uribe es una pequeña isla que tiene una forma redondeada de 1,150 metros de diámetro. El pueblo se ubica al noroeste a unos $25 \mathrm{~km}$ del litoral del Océano Pacifico, en el lago de Mextliapan que alimenta el río San Pedro (México,2005). Desde el continente está a $36 \mathrm{~km}$ de la municipalidad de Santiago Ixcuintla, a la que pertenece administrativamente, y a $98 \mathrm{~km}$ de la ciudad de Tepic, la capital del estado de Nayarit. Específicamente, la isla se encuentra al norte de la Riviera Nayarit10 y en medio a la región de Marisma Nacional11, bajo pantanos, manglares y

\footnotetext{
${ }^{10}$ La costa de la Riviera Nayarit se extiende por los municipios de Bahía de Banderas, Compostela, San Blas, Santiago Ixcuintla y Tecuala.

${ }_{11}^{11}$ La Reserva de la Biosfera Marismas Nacionales, que está ubicada en los municipios de Acaponeta, Rosamorada, Santiago Ixcuintla, Tecuala y Tuxpan, en el Estado de Nayarit, fue creada mediante el Decreto Presidencial publicado en el Diario Oficial de la Federación el 12 de mayo del 2010. Así Mexcaltitan de Uribe es denominada como una subzona de asentamientos humanos, en la que se permite actividades de acuacultura, pesca, silvicultura, turismo y turismo de bajo impacto ambiental, entre otras. [VéaseloMéxico - Diario Oficial de la Federación (2013). Resumen del Programa de Manejo del Area Natural Protegida con el carácter de Reserva de la Biosfera Marismas Nacionales Nayarit. Consultado en mayo $1, \quad 2015, \quad$ de http://dof.gob.mx/nota detalle.php?codigo=5294348\&fecha=03/04/2013]. Junto con el ecosistema de manglar de Chiapas, la región de Marismas Nacionales de Nayarit compone los ecosistemas de este tipo más importantes del Pacífico de América (México 2005).
} 
canales. El acceso a la isla es por lancha desde el embarcadero La Batanga distante a $6 \mathrm{~km}$ de Mexcaltitán.

Por la limitación geográfica, es decir, las dimensiones del territorio, no hay mucho cambio respecto a la demografía del pueblo. En 1950 la población de la isla era de 932 habitantes (Gálvez-González, s.d.).De acuerdo con los datos del Instituto Nacional de Estadística y Geografía - INEGI, en el 2005 la población de la isla era de un total de 895 de habitantes, mientras que en el 2010 bajo a un total de 818 habitantes (tabla 1) (México, 2015).

Con respecto a los indicadores de carencia en viviendas en el censo de 2010 (tabla 2) se ha identificado que del total de las viviendas particulares del pueblo $7(3.04 \%)$ no tiene drenaje, 3 $(1.30 \%)$ no tiene luz eléctrica, 38 (16.52\%) no tiene agua entubada y $13(5.65 \%)$ no tiene sanitario (México, 2015).

Tabla 2. Indicadores de carencia en viviendas

\begin{tabular}{|c|c|c|c|c|}
\hline \multirow{2}{*}{ Indicadores } & \multicolumn{2}{|c|}{$2005^{[1]}$} & \multicolumn{2}{|c|}{$2010^{[2]}$} \\
\hline & Valor & $\%$ & Valor & $\%$ \\
\hline $\begin{array}{l}\text { Viviendas particulares } \\
\text { habitadas }\end{array}$ & 229 & & 230 & \\
\hline Viviendas con piso de tierra & 14 & 6.11 & 2 & 0.87 \\
\hline $\begin{array}{l}\text { Acceso a los servicios } \\
\text { básicos en las viviendas } \\
\text { particulares habitadas }\end{array}$ & & & & \\
\hline Viviendas sin drenaje & 19 & 8.30 & 7 & 3.04 \\
\hline Viviendas sin luz eléctrica & 2 & 0.87 & 3 & 1.30 \\
\hline Viviendas sin agua entubada & 14 & 6.11 & 38 & 16.52 \\
\hline Viviendas sin sanitario & 19 & 8.30 & 13 & 5.65 \\
\hline
\end{tabular}

\section{Notas:}

[1] datos del II Conteo de Población y Vivienda 2005. INEGI. [2] datos del Censo de Población y Vivienda 2010: Principales Resultados por Localidad. INEGI

Fuente: adaptado de México (2015).

Con respecto a los indicadores de escolaridad se destaca que entre 2005 e 2010 la población de 15 años o más analfabeta ha disminuido levemente del $5.68 \%$ a uno $5.58 \%$, mientras la población de 6 a 14 años que no asiste a la escuela ha aumentado del $0.57 \%$ a uno $0.87 \%$. Todavía el promedio de $51.83 \%$ de la población de 15 años y más con educación básica incompleta se mantiene muy alta en 2010, a pesar de que ha reducido desde el conteo de 2005 que registro uno 52.84\% para este indicador (tabla 3).

Tabla 3. Indicadores de escolaridad

\begin{tabular}{|l|c|c|}
\hline Población total & $\mathbf{2 0 0 5}$ & $\mathbf{2 0 1 0}$ \\
\hline \% de población de 15 años o más analfabeta & 5.68 & 818 \\
\hline $\begin{array}{l}\text { \% de población de 6 a 14 años que no asiste a } \\
\text { la escuela }\end{array}$ & 0.57 & 0.87 \\
\hline $\begin{array}{l}\text { \% de población de 15 años y más con } \\
\text { educación básica incompleta }\end{array}$ & 52.84 & 51.83 \\
\hline
\end{tabular}

Fuente: adaptado de México (2015). Estimaciones del CONEVAL, con base en INEGI, II Conteo de Población y Vivienda 2005 y la ENIGH 2005. Estimaciones de CONEVAL con base en el Censo de Población y Vivienda 2010.

El índice de marginación del pueblo que era -1.08313 en 2005, calificado de bajo grado, ha pasado en 2010 a -0.91747 , o sea a un medio grado. Estos resultados sitúan la isla Mexcaltitlán en la posición 90,056 en el contexto nacional (tabla 4).

Tabla 4. Indicadores de marginación

\begin{tabular}{|l|c|c|}
\cline { 2 - 3 } \multicolumn{1}{c|}{} & $\mathbf{2 0 0 5}$ & $\mathbf{2 0 1 0}$ \\
\hline Índice de marginación & - & - \\
\hline Grado de marginación & 1.08313 & 0.91747 \\
\hline $\begin{array}{l}\text { Lugar que ocupa en el contexto } \\
\text { nacional }\end{array}$ & Bajo & Medio \\
\hline
\end{tabular}

Fuente: adaptado de México (2015), con base en los estimaciones del CONAPO, los índices de marginación 2005; y CONAPO (2011).

Por fin se destaca que la principal actividad económica del pueblo es la pesca del camarón.

El Estado de Nayarit, donde se encuentra la isla, también presenta severos problemas con la vulnerabilidad social. Según datos de CONEVAL (2012), la población en situación de pobreza en 2010 era de $41.4 \%$ aumentando para $47.6 \%$ en 2012. De esos, en $2010,8.3 \%$ estaban en situación de pobreza extrema pasando para $11.9 \%$ en 2012. Conforme otro indicador de CONEVAL, $74.8 \%$ de la población sufre con al menos una carencia social; dato que ascendió para $75.8 \%$ en 2012 . La población con ingreso inferior a la línea de bienestar mínimo en 2010 era de 17.8\%, y en 2012, 23.4\%.

\subsection{Turismo en Mexcaltitlán y el Programa Pueblos Mágicos}

La importancia turística de la isla se debe a su historia prehispánica. Se cree que Mexcaltitán de Uribe sea la legendaria Aztlán, el lugar de donde partieron las tribus aztecas que asiéntanse en el Valle de México, donde se ha fundado la ciudad de Tenochtitlán. Por eso la isla es denominada la "cuna de la identidad mexicana"12.

El pueblo tiene una plaza central que data del siglo XVII, una iglesia dedicada a San Pedro y San Pablo, además de un pequeño museo llamado "El origen". Además en la temporada de lluvias, de junio a octubre, las calles se llenan del agua, se volviendo en canales navegables, haciendo que haya que cruzarlas en botes. Por esa característica la isla es llamada de "La Venecia mexicana".

Debido a la estructura urbana en forma de un gran óvalo, con la traza de sus calles de norte a sur y de oriente a poniente, además de su importancia para la historia social, política y urbana de México, la isla ha recibido el nombramiento de Zona de Monumentos Históricosen el 1986 (México, 1986).El decreto publicado en el Diario Oficial de la Federación el 8 de diciembre del 1986, dice que por la acelerada transformación de la fisonomía y del medio ambiente de la isla y del pueblo de Mexcaltitán, es urgente la protección de su trazo urbano, a través de la restauración de edificios, de la regulación e integración de nuevas

\footnotetext{
${ }^{12} \mathrm{~A}$ pesar de que no ha comprobación sobre el origen acerca del cambio de pueblo de Mexcaltitlán al valle de México la comparación entre las fotografías aéreas de Mexcaltitán y los dibujos del siglo XVI hecho por los conquistadores españoles de Tenochtitlán, muestra que ambos lugares tienen el mismo trazado de calles y canales, estableciendo una semejanza entre ambas zonas. (Véaselo http://mexico.pueblosamerica.com/i/mexcaltitan-de-uribe/,_consultado en mayo 1, 2015)
} 
construcciones, de la dotación de los indispensables servicios públicos, además de la necesaria protección del medio ambiente (México, 1986, México, 2005).

Por su cultura y tradiciones características Mexcaltitán fue declarada por el gobierno federal como "Pueblo Mágico" en el 3 de septiembre del 2001, con el potencial de enfocarse en turismo cultural y ecoturismo para promover el crecimiento de la zona (México, 2011b).

Todavía, desde 2005 este nombramiento se vio amenazado por el argumento de "la falta de aplicación del apoyo oficial destinado por el gobierno municipal a obras y servicios necesarios para permanecer en el programa"(México, 2011b). Como una tentativa de mantener el pueblo en el Programa, en el 11 de febrero de 2009 se solicitó la ampliación del periodo de reserva del 01 de abril del 2006 al 01 de abril del 2011, con el fin de que se pondría en riesgo la economía estatal y el desarrollo del Plan Maestro de Desarrollo Urbano y Turístico de la isla de Mexcaltitán de Uribe13.

Entretanto, en julio del 2009 el Comité Interinstitucional de Evaluación de Pueblos Mágicos retiró Mexcaltitán de Uribe de la lista del programa bajo laexplicación de que no se había cumplido de compromisos de limpieza y del ordenamiento del ambulantaje por parte del gobierno municipal14(México, 2011b, Mejía González, 2011).

Considerando la exposición presentada en ese trabajo se defiende que para que el pueblo ajústese a las exigencias del PPM sería necesario que un conjunto de proyectos simultáneos fueran llevados a cabo, desde la sensibilización de la población para la posibilidad de trabajar con el turismo, hasta su capacitación para promoverlas actividades necesarias. Por otro lado, para que eso ocurriera sería preciso no solo proyectos de capacitación, sino un condiciones socio estructurales básicas.

De acuerdo con el argumento propuesto se busca elucidar la perspectiva de actuación del Estado federal manifestada en un programa de turismo, que se ha repercutido en una práctica de segregación de espacios y de desapropiación del patrimonio cultural, a partir del uso de las transferencias condicionadas.

Cumple mencionar que la responsabilidad por la pérdida del nombramiento, fue colocada en los habitantes, los cuales se considera que no se empeñaran o trabajaran para cumplir los criterios de orden y calidad establecidos para mantenerse en el programa. Hay también menciones de conflictos de interés político como factor de la pérdida del título. Dicho de otra forma se ha identificado una justificación social por el fracaso del programa en la isla, a través de la criminalización de los habitantes, por su capacidad insuficiente o por parecieren "sucios" además de otros argumentos y juicios a-contextuales que poco comportan los demás factores coyunturales de la isla.Ese escenario incurrió en una perspectiva que penaliza la comunidad local e incrementa las dificultades sociales enfrentadas, ya que por esa condición el

${ }^{13}$ Véaselo en

${ }^{14}$ El senador Nayarita Raúl Mejía presentó una solicitud al Senado de la República, para reconsiderarse la isla dentro del programa de "Pueblos Mágicos" y así impulsar el turismo de la zona, todavía no se logró éxito.
Estado legitima su no intervención en la comunidad sobe la eje del no cumplimiento de requisitos para la transferencia. El efecto es un rechazo del turismo cuanto un producto endógeno, además del abandono de una región de grande relevancia histórica.

Este mismo aspecto puede ser identificado a partir de los datos presentados por Coneval $(2015,2010)$, o no, de la isla. El Indicador de Rechazo Social (IRS) de esta localidad en 2000 fue de -1.216178 considerado "Bajo", ocupando la posición de 92.624. En 2005, ya como Pueblo Mágico, la condición de la isla fue para "muy bajo" reduciendo su indicador para -1.3875. En 2010, con reciente pérdida del título, el rango era "muy bajo" y la posición fue para 97.131 indicando un mejoramiento en la calidad de vida de la población. Entretanto, a partir de eso período, ya no se presenta datos estratificados de la isla considerando solamente los efectos a nivel de municipios, en el caso, Santiago de Ixcuintla. De igual forma se observa cambios de datos sobre la evaluación de la pobreza en Nayarit. Considerando que en 2008 la población en extrema pobreza era de $6.4 \%$, en 2010 fue de $8.2 \%$ y en 2012 de $11.9 \%$. Estos datos oficiales del gobierno contrastan con otros, en el mismo documento, que oscilan en 2010 con datos de $8.2 \%$, $7.6 \%$ o $9.6 \%$ y en 2012 entre $11.9 \%$ y $10.7 \%$.

También se desataca que las muestras de arqueología prehispánica local fueron llevadas a otros museos por considerarse que no había seguridad para su exposición en Mexcaltitán. Se presentando una situación de evasión de material arqueológico e histórico, substituidos por réplicas, por la fragilidad en la organización del turismo del lugar.

\section{Consideraciones finales}

El objetivo fue problematizar las interferencias de las políticas públicas que tiene como característica el uso condicionado de las transferencias en la conformación de espacios de segregación. Argumentando sobre un proceso de cambio en los fundamentos de las políticas, ese trabajo se desprendió en observar las incoherencias del Programa de Pueblos Mágicos a partir de la experiencia de intervención del turismo en la isla Mexcaltitlán de Uribe, en Nayarit, México.

EI PPM, por su carácter de asistencia condicionada y fuerte orientación a la iniciativa privada operó el rechazo de una zona de monumento histórico en búsqueda de la rentabilidad inmediata. EI resultado fue un proceso de segregación sociopolítica formalizando la no intervención gubernamental; evasión del patrimonio cultural; y en el lugar de una acción supuestamente articulada por el Estado, la isla fue rechazada (sobrepenalizada) por sus propias condiciones de vulnerabilidad social, económica estructural.

Es importante considerar que frente la crisis del Estado de Bienestar social, las políticas públicas mexicanas, como de otros países de Latinoamérica, presentan dispositivos que buscan atender sectores determinados o específicos de la sociedad. Este fenómeno, que cambia la lógica de igualdad de posiciones para igualdad de oportunidades, legitima programas estatales de 
transferencias condicionadas en el país, fundamentado por una individualización del bienestar que ha terminado por justificar la desigualdad social basada en la meritocracia. La consecuencia es la manifestación de una sociedad fragmentada y una segregación espacial y social atenuada por los problemas sociales anteriormente ya imperantes en la localidad.

\section{Referencias}

Abramovay, P. V. yMalaguti, V. (org.). (2010). Seminário Depois do Grande Encarceramento. Rio de Janeiro: Revan.

Balslev-Clausen,H. y Alberto-Velázquez, M. (2013). El turismo cultural en Argentina, México y Brasil. Avances y desafíos. Diálogos Latinoamericanos, Latin American Center, University of Aarhus, 21, 9-28.

Boltvinik, J.(2013). Necesidad de reformar la reforma social neoliberal implantada en México a partir de los años noventa. En Alternativas en la crisis para la transformación de las políticas sociales, CIAD (Centro de Investigación en Alimentación y Desarrollo, A.C.).

Carvalho, F. C. C. (2014) Análise das Influências Internacionais sobre as Políticas Públicas de Turismo no Brasil. In VI Congreso Latinoamericano de Investigación Turística. Neuquén: Argentina.

Castel, R. (2014) Los riesgos de exclusión social en un contexto de incertidumbre. En Revista Internacional de Sociología (RIS), 72(1), 15-24, DOI:10.3989/ris.2013.03.18

Consejo Nacional de Evaluación de la Política de Desarrollo Social (CONEVAL). (2012) Medición de la Pobreza, Nayarit, 2012. Acceso en 15 de Mayo de 2015. Disponible http://www.coneval.gob.mx/Informes/Coordinacion/PDFS_TABLAS_POBRE ZA_2012/Nayarit\%202012.pdf

Dubet, F. (2012). Os limites da igualdade de oportunidade. Cadernos Cenpec,São Paulo, 2, (2), 171-179

Esping-Anderson, G. (1993). Los tres mundos del estado del bienestar. EdicionsAlfons el Magnànim. Institució Valencia D'estudis I Investigació Generalitat Valenciana, Diputació Provincial de València.

Huesca, L; Aranda, P.; Hobarth, J. y Valencia, V. (2012). Evolución de la pobreza en México y en el Distrito Federal, 1992-2010. Valorización crítica de las metodologías de medición, las fuentes y las interpretaciones In Ordóñez, Gerardo Barba (cord). La pobreza Urbana en México: nuevos enfoques y retos emergentes para la acción pública. Juan Pablos Editor, 2390.

Gálvez-Gonzáles, L.A. (s.d.)El patrimonio cultural. Las zonas de monumentos históricos. Consultado en mayo 1, 2015 de http://biblioteca.diputados.gob.mx/janium/bv/ce/scpd/LX/patri_cultu_Ix.pdf International centre for Prision Studies - ICPS.(2014).Walmsley, R.WorldPrision Population List.TenthEdition, University of Essex, UnitedKingdom.

Jusidman, C.(2012). Contextos urbanos, pobreza y violencia. In Ordóñez, Gerardo Barba. La pobreza Urbana en México: nuevos enfoques y retos emergentes para la acción pública. Juan Pablos Editor, 361-388.

Hernández López, J. J. (2009). Tequila: Centro mágico, pueblo tradicional. ¿Patrimonización o privatización? Andamios. Revista de Investigación Social, Universidad Autónoma de la Ciudad de México Distrito Federal, México,6(12), 41-67.

Martinez, M. (2010). Populismo punitivo, maiorias e vítimas. In: Abramovay, P. V; y Malaguti,V. (org.). Seminário Depois do Grande Encarceramento. Rio de Janeiro: Revan.
Mejía González, R. (2011). Proposición con Punto de Acuerdo por el que se exhorta al titular del Poder Ejecutivo para que a través de la Secretaría de Turismo, se reconsidere reintegrar a la Isla Mexcaltitán al Programa de Pueblos Mágicos.Salón de Sesiones del Senado de la República, D.F., a 18 de octubre del 2011.

México. (1986). Decreto por el que se declara una zona de monumentos históricos en la población de Mexcaltitán de Uribe, Municipio de Santiago Ixcuintla, Nay. Publicado en 08 de diciembre de 1986. Instituto Nacional de Antropología e Historia. Consultado en mayo 1, 2015 de http://www.ordenjuridico.gob.mx/listDependencia.php?idEst=98\&poder=ejec utivo\&liberado $=\mathrm{si}$

México (2011a).ACUERDO por el que se establecen los Lineamientos generales para la incorporación y permanencia al Programa Pueblos Mágicos.Diario Oficial (Primera Sección). Viernes 26 de septiembre de 2014.

México (s/d).Guía de incorporación y permanencia. Pueblos Mágicos. Secretaria de Turismo, SECTUR, Consultado en mayo 1, 2015 de www.sectur.gob.mx/wp-content/.../GUIA-FINAL.pdf

México (2005). Estudio previo justificativo para el establecimiento del área natural protegida. Área de protección de flora y fauna marismas nacionales Nayarit. Secretaría de Medio Ambiente y Recursos Naturales, Comisión Nacional de Áreas Naturales Protegidas.

México, Gobierno de la Nación. (2013). Plan Nacional de Desarrollo $2013-$ 2018. Diario Oficial.

México, Comisión de Turismo de la LXI Legislatura de la Cámara de Senadores del $\mathrm{H}$. Congreso de la Unión. (2011b).De la Comisión de Turismo, el que Contiene Punto de Acuerdo: que exhorta a la Titular de la Secretaría de Turismo del Gobierno Federal a Reincorporar la Isla de Mexcaltitán, Nayarit, al Programa Pueblos Mágicos. 24 de octubre del 2011. Consultado en mayo 1, 2015.

México.(2015b).Sistema de Apoyo para la Planeación del PDZP. Catálogo de Localidades. Secretaría de Desarrollo Social, Unidad de Microrregiones. Consultado en mayo 1, 2015, de http://www.microrregiones.gob.mx/catloc/contenido.aspx?refnac $=180150038$

Montagut, Teresa (2004). Política Social: Una introducción. Ariel Sociología. Ed.2

Moreno-Brid, J. C.; Bosch (2014).Desarrollo y crecimiento en la economía mexicana: Una perspectiva histórica, Fondo de Cultura Económica, México.

North, Douglass C. (1993:2012). Instituciones, Cambio institucional y desempeño económico. Trad. Agustín Bárcena, México, FCE.

Rusche, G.,Kirchheimer, Otto. (2004) Punição e Estrutura Social. 2 ed., Instituto Carioca de Criminologia, Pensamento criminológico n.3, Revan.

Vite Pérez, M. A. (2014). México, ¿Hacia la consolidación de un modelo de igualdad de oportunidades para una sociedad fragmentada? Perfiles Latinoamericanos, (44), pp. 33-57. Facultad Latinoamericana de Ciencias Sociales, Distrito Federal, México. Wacquant, L.2011. As prisões da miséria. 2.ed: Rio de Janeiro: Zahar.

Wacquant, L. (2012). A política punitiva da marginalidade: revisitando a fusão entre "workfare" e "prisonfare, Epos (Rio de Janeiro), pp. 1-28; also in *Brazilian volume (editedby Carlos Jobim).

Consejo Nacional de Evaluación de la Política de Desarrollo Social (Coneval). Indicadores de pobreza extrema y carencia por acceso a la alimentación por municipio 2010Coneval, 2015. Disponible en:http://www.coneval.gob.mx/Medicion/Paginas/Indice-de-rezago-social2005.aspx. Consultado en mayo 31, 2015.

Consejo Nacional de Evaluación de la Política de Desarrollo Social (Coneval).Grado de Rezago Social por AGEB urbana, nacional 2010. Disponible en:http://www.coneval.gob.mx/Medicion/Paginas/Indice-derezago-social-2005.aspx. Consultado en mayo 31, 2015. 